\title{
Resúmenes de los trabajos presentados en la XI Reunión Anual de la Red Cochrane Iberoamericana 2012, XIII Congreso LatinCLEN. 23 - 25 de mayo de 2012. Lima, Perú.
}

\section{Costo-efectividad de Palivizumab en profilaxis de infección de virus respiratorio sincicial en pacientes de alto riesgo en Chile.}

Soto D, Vallejos C, De La Puente C, Velázquez M.

Centro de Excelencia CIGES, Universidad de La Frontera; Temuco, Chile.

Introducción: En niños de riesgo, las infecciones por virus respiratorio sincicial (VRS) producen un elevado consumo de recursos sanitarios. El Palivizumab es efectivo en disminuir la probabilidad de hospitalización por VRS, sin embargo su elevado costo impide utilizarlo ampliamente. Objetivo: Evaluar la costo-efectividad del Palivizumab como profilaxis VRS en diferentes grupos de riesgo en Chile. Metodología: Se elaboran 4 árboles de decisión, comparando el uso de Palivizumab como profilaxis, versus su no uso, en los siguientes 4 grupos: prematuros con displasia broncopulmonar (DBP); prematuros $<32$ semanas gestación; prematuros entre 32-35 semanas de gestación; y los tres grupos anteriores simultáneamente. Se usa la perspectiva del proveedor (Ministerio de Salud), y horizonte temporal de un año. La medida de efectividad fueron las hospitalizaciones evitadas (HE). Los costos se estiman a partir de datos nacionales. Se realiza análisis de sensibilidad de una vía considerando: efectividad Palivizumab, días de hospitalización, costo de Palivizumab. Resultados: La efectividad del Palivizumab es diferente en los grupos de riesgo. Al realizar profilaxis con Palivizumab, la razón incremental de costo efectividad (ICER) en los diferentes grupos fue: en los tres grupos en forma simultánea CH $\$ 22.696 .430 / \mathrm{HE}$, DBP CH\$43.948.779/HE, prematuros < 32 semanas $\mathrm{CH} \$ 21.855 .955 / \mathrm{HE}$, prematuros entre $32-35$ semanas CH\$8.560.693/HE. El grupo que resulto más costo efectivo (menor ICER) fue en prematuros entre 32-35 semanas de gestación. Conclusión: No es recomendable la realización de profilaxis con Palivizumab en prematuros $<32$ semanas de gestación, en pacientes con DBP ni el realizar profilaxis a los tres grupos de riesgo simultáneamente; sin embargo, se debe evaluar la realización de profilaxis en el grupo de prematuros de 32 a 35 semanas. El proveedor (MINSAL) debe definir lo que se estaría dispuesto a pagar.

\section{Análisis costo efectividad del stent farmacológico vs stent} no farmacológico en cardiopatía isquémica, en Chile.

De la Puente C, Vallejos C, Soto D, Velásquez M.

Centro de Excelencia CIGES, Universidad de La Frontera; Temuco, Chile.

Antecedentes: Los altos costos del stent farmacológico en Chile generan inquietudes respecto a su costo-efectividad. Existe escasez de estudios de este tipo en la literatura nacional. Objetivos: Evaluar y comparar los costos y la efectividad de dos alternativas de stent, farmacológico y no farmacológico. Material y métodos: Análisis costo-utilidad basado en un modelo de Markov con datos provenientes de una cohorte de 263 pacientes con diagnóstico de cardiopatía isquémica intervenidos en el hospital Las Higueras de Talcahuano, Chile, con 426 stents implantados, de los cuales $14,8 \%$ fueron farmacológicos y $85,2 \%$ no farmacológicos. La medida de efectividad fue la tasa de reestenosis y el tiempo de reestenosis. Los resultados de efectividad se expresan en años de vida ajustados por calidad (QALYs) ganados. Los costos, expresados en moneda nacional 2011, fueron calculados a partir de los datos de la cohorte estudiada. La perspectiva de evaluación fue desde el presupuesto público de salud. Se modela una cohorte desde los 63 años a los 80 años, expectativa de vida chilena. Se aplica tasa de descuento de $0,3 \%$ y $6 \%$ para resultados y costos. Se realiza análisis de sensibilidad de los costos, los valores de utilidad y de las variables de transición entre los estados. Resultados: No hubo diferencias en la tasa de reestenosis entre ambos stents, aunque sí hubo diferencias en el tiempo de reestenosis. La razón de costo efectividad incremental (RCEI) sin tasa de descuento fue de CHI\$235.749 por QALY ganado al utilizar el stent farmacológico, valor por debajo del equivalente a 1 Producto Interno Bruto per cápita para el año 2011 en Chile. Conclusiones: El stent farmacológico es muy costo efectivo, con respecto al stent no farmacológico. La RCEI no se ve afectada por el análisis de sensibilidad (variabilidad de costos, rangos de utilidad utilizados, probabilidad de reestenosis).
02. La eficacia de dos métodos de muestreo de Papanicolaou: un ensayo controlado aleatorio.

Aleman $\mathbf{A}^{1}$, Reyes $\mathbf{I}^{1}$, Solazzo $\mathrm{A}^{1}$, Stoll $\mathbf{M}^{1}$, Bonilla Silvia ${ }^{2}$, Casserta $\mathbf{B}^{2}$, De los Santos $\mathrm{H}^{2}$.

Departamento de Medicina Preventiva de la Facultad de Medicina, Universidad de la República; Montevideo, Uruguay

Intendencia de Montevideo, Uruguay

Antecedentes: El cáncer cervical es la tercera causa de mortalidad por cáncer en mujeres en el mundo. El examen del cuello uterino (Papanicolau) ha demostrado ser eficaz para disminuir la incidencia de la enfermedad invasiva mediante la detección de anomalías precancerosas del cuello uterino. Eficacia de la prueba puede verse afectada por el diseño del dispositivo de muestreo. En algunos de los servicios de atención públicos de Uruguay el método de extracción de muestras es utilizando una varilla fina de madera que presenta un costo sensiblemente menor que el citocepillo. Objetivo: Evaluar la eficacia de uso de la varilla de madera vs citocepillo como dispositivos de muestreo de estudios de Papanicolaou. Metodología: Este es un estudio aleatorizado y controlado simple ciego con enmascaramiento de la asignación que evaluó la presencia de células endocervicales en frotis de Papanicolaou realizadas con varilla de madera o citocepillo. Resultados: Se incluyeron 511 mujeres que se asignaron al azar a citocepillo o al método de muestreo con varilla para la prueba de Papanicolaou. Los grupos presentaron características comparables. Los resultados muestran que las células endocervicales se encontraban más frecuentemente presentes en las muestras con citocepillo que con varilla de madera (RR 1,55 IC95\% 1,075-2,25). Conclusión: Citocepillo debe ser considerado como el método más adecuado para la toma de muestras de frotis de Papanicolaou en todos los ambientes públicos de Uruguay con el fin de evitar la inequidad en la calidad de la atención e inconvenientes para las mujeres.
04. Ensayo clínico aleatorio del efecto del consumo de cacao en parámetros cardiovasculares e inmunológicos en pacientes colombianos con diagnóstico reciente de hipertensión arterial estadio 1.

Giraldo M, Jaramillo CJ, García HI, Alcaraz G, Patiño P, Angarita LA. Universidad de Antioquia; Medellín, Colombia.

Antecedentes: Adoptar estilos de vida saludable busca prevenir elevaciones de presión arterial (PA), disminuir el riesgo cardiovascular y mejorar la eficacia de tratamientos farmacológicos. El cacao es rico en flavanol, que incrementa la biodisponibilidad de óxido nítrico. Tres metanálisis de poblaciones anglosajonas han mostrado que el cacao reduce la HTA. Objetivo: Estimar el efecto de consumo de cacao comparado con placebo en la PA, parámetros cardiovasculares e inmunológicos en individuos con diagnóstico nuevo de HTA esencial estadio 1. Material y métodos: Ensayo clínico paralelo, de superioridad, en dos grupos, uno de intervención consumió 50 gr. de chocolate con $70 \%$ de sólidos de cacao y el placebo comió chocolate exento de sólidos de cacao durante 12 semanas. Se hizo asignación aleatoria con ocultamiento de la secuencia y enmascaramiento del evaluador del desenlace y del estadístico hasta la finalización del análisis. El desenlace principal fue disminución de $4 \mathrm{mmHg}$ en la PA diastólica medida por monitorización ambulatoria de presión arterial ( 24 horas). Las diferencias entre los grupos se evaluaron con un análisis de covarianza ajustando por la PA inicial. Se realizó análisis por intención a tratar. Resultados: La PAD disminuyó en ambos grupos luego de 12 semanas con una diferencia a favor del grupo de chocolate negro $(0.70 \mathrm{~mm} \mathrm{Hg})$, la cual no se encontró al ajustar por el valor inicial. No hubo diferencias en los parámetros inflamatorios y perfil lipídico, si en el porcentaje de dilatación de la arteria braquial. Conclusiones: El consumo de 50 gr. diarios de chocolate negro 84 días no mostró efecto en cifras de PAD, en parámetros cardiovasculares e inflamatorios. Introducir adecuadamente el chocolate en la alimentación de sujetos con diagnóstico de HTA estadio 1 no tiene efectos negativos ni implicaciones clínicas de importancia en la composición corporal y los cambios en el perfil lipídico. 


\section{Costo-efectividad del Tratamiento del Síndrome de Déficit Atencional en Chile.}

Velásquez M, Vallejos C, De La Puente C, Soto D.

Centro de Excelencia CIGES, Universidad de La Frontera; Temuco, Chile

Antecedentes: El Síndrome Déficit Atencional (SDAH) es diagnosticado a edad escolar. El tratamiento farmacológico (Metilfenidato) y psicosocial es fundamental para el buen desarrollo a nivel escolar y social de los pacientes, y su efectividad ha sido demostrada. Para el sistema de Salud de Chile es de suma relevancia conocer los costos y resultados de la intervención en esta patología. Objetivos: Determinar la costo-efectividad del tratamiento farmacológico y psicosocial del SDAH en Chile para pacientes diagnosticados a partir de los 6 años de edad. Material y métodos: Un modelo de Markov con 4 estadios fue construido para simular la historia natural (No tratamiento) y el tratamiento de una cohorte hipotética de 10.000 individuos diagnosticados con SDAH desde los 6 años de edad hasta los 18 años. Los costos fueron medidos en pesos chilenos 2011, y los resultados se expresan en años de vida ganados ajustados por calidad (QALY). Para estimar el valor de utilidad se utiliza la metodología Delphi, usando datos de otros estudios. Se aplican tasas de descuento de $0 \%, 3 \%$ y $6 \%$. Se realiza análisis de sensibilidad a costos, valores de utilidad, y probabilidades de transición. Resultados: El tratamiento para el SDAH tiene un costo total promedio ponderado por paciente de CH $\$ 2.471 .959$, con una utilidad de 12,9 QALY, a una tasa de descuento del $0 \%$. La razón de Costo-efectividad incremental (ICER) es de CH\$1.691.183, la cual está por debajo de 1 Producto Interno Bruto per cápita en Chile, lo que la sitúa como una intervención muy costo-efectiva. Conclusiones: El tratamiento farmacológico y psicosocial del SDAH es una estrategia muy costo-efectiva desde la perspectiva de los Sistemas de Salud, por lo cual parece muy recomendable su adopción integral en vistas a lograr un cabal desempeño en las distintas áreas de desarrollo del niño en etapa escolar.

\section{Análisis de costo/efectividad en Colombia de los tratamientos antimicrobianos para la vaginosis bacteriana en mujeres sintomáticas no gestantes.}

Díaz M, Páez C, Ruíz A, Díaz J, Prieto V, Urrego J, Gaitán H.

Universidad Nacional de Colombia.

Antecedentes: existen diferentes recomendaciones para el tratamiento de la vaginosis bacteriana, sin embargo, no existe evidencia en nuestro país de la costo/efectividad de estas tecnologías. Objetivo: estimar la costo/efectividad en Colombia del uso de metronidazol oral en multidosis, metronidazol intravaginal (gel) y clindamicina oral e intravaginal (crema), comparado con metronidazol oral en monodosis, para el tratamiento de la vaginosis bacteriana en mujeres sintomáticas no gestantes. Metodología: estudio de costo/efectividad, basado en un modelo de árbol de decisiones, en mujeres sintomáticas no gestantes, con diagnóstico clínico de vaginosis bacteriana. Se consideró un horizonte temporal de un mes. Desde la perspectiva del tercer pagador, se estimaron los costos directos médicos en pesos colombianos de 2011, empleando el manual tarifario SOAT y el SISMED. La efectividad se definió como mejoría clínica. Las probabilidades del modelo se extrajeron de la literatura. Las preferencias para los estados de salud se determinaron mediante consulta a un grupo de expertos. Se estimaron las razones de costo/efectividad promedio e incremental, y se realizó un análisis de sensibilidad determinístico univariado para los costos y probabilidades de eventos clínicos, usando el programa TreeAge Pro Suite ${ }^{\mathbb{R}}$. Resultados: todas las alternativas fueron dominadas por clindamicina oral. Esta tecnología obtuvo un costo promedio por caso de mejoría clínica de Col\$2.271, representando un ahorro extra de Col\$ 10.844 por caso de mejoría clínica extra en comparación con metronidazol oral en monodosis. Los resultados fueron robustos a variaciones de los parámetros del modelo Conclusiones: clindamicina oral (300 miligramos, dos veces al día, durante 7 días) representa en Colombia la mejor alternativa en términos de costo/efectividad, para el tratamiento de la vaginosis bacteriana en mujeres sintomáticas no gestantes.

\section{Eficacia y efectividad de la provisión de alimentos complementarios en la prevención de la desnutrición en la población Infantil.} Fiestas-Saldarriaga FA, Aparco-Balboa JP, Bravo-Rebatta F, Suarez V.
Instituto Nacional de Salud.

Antecedentes: En el Perú hay regiones con más de 30\% de niños desnutridos. La provisión de alimentos complementarios (AC) ha sido una medida ampliamente usada para hacer frente a esta situación. Objetivo: Evaluar la evidencia científica sobre la eficacia o efectividad de programas de provisión de $\mathrm{AC}$ para la prevención primaria o secundaria de la desnutrición en niños de 6 a 36 meses. Métodos: Se realizó una sinopsis de revisiones sistemáticas y meta-análisis, con búsquedas en Medline, Embase y LILACS. El tipo de intervención evaluada es el suministro regular de $\mathrm{AC}$, específicamente alimentos ricos en energía y proteínas. Las intervenciones de comparación incluyen la educación alimentaria (EA), placebo, el status quo u otras orientadas a mejorar el estado nutricional de la población infantil. Las variables resultado incluyeron peso y la talla. Resultados: Se identificó 3701 artículos. Se seleccionaron por título y resumen 13 artículos para evaluación a texto completo, de los cuales 4 fueron incluidos a la sinopsis. Tres de estas revisiones sistemáticas tienen resultados que apoyan la hipótesis que la adición de AC un efecto positivo en el peso y talla comparado con otro tipo de intervenciones diferentes a la EA. Comparado con la EA, los AC no muestran superioridad clara. La combinación de AC y EA tiene un efecto mayor que cuando se aplican estas intervenciones por separado. La adición de AC tiene un efecto más claro cuando se aplica en lugares donde existe inestabilidad alimentaria, como es el caso de poblaciones muy pobres. En condiciones más estables de accesibilidad a alimentos, la EA mostró ser tan eficaz y efectiva que la adición de AC. Por otra parte, una revisión sistemática que evaluó la eficacia de proveer AC en ensayos clínicos en infantes desnutridos, no encontró beneficio de esta intervención frente a placebo. Conclusión: La provisión de AC y la EA muestran ser efectivas para la prevención primaria de desnutrición en niños de 6 a 35 meses, aunque el efecto es pequeño si son implementadas por separado, mejorando si se aplican en combinación. Respecto a prevención secundaria, la eficacia de únicamente adicionar alimentos complementarios es aún controversial.

\section{Antibiótico profiláctico en pacientes sometidos a cistoscopía con orina estéril: Un experimento clínico controlado doble ciego.}

García-Perdomo HA, López H, Carbonell J, Castillo D, Cataño JG, Serón P. Escuela de Medicina de la Facultad de Salud, Universidad del Valle; Cali, Colombia.

Objetivo: Establecer la eficacia de la utilización de antibiótico profiláctico previo a la cistoscopia en pacientes ambulatorios en disminuir la incidencia de infección urinaria post procedimiento. Metodología: Se realizó un experimento clínico aleatorizado con doble enmascaramiento, multicéntrico, en pacientes mayores de 18 años que son sometidos a cistoscopia por cualquier indicación no urgente (ambulatoria) con resultado de urocultivo negativo previo al procedimiento, en un hospital o clínica de tercer nivel de atención o una clínica especializada en urología. Se estableció un tamaño de muestra mínimo de 136 pacientes en cada brazo para obtener diferencias que no fueran debidas al azar. Los pacientes fueron asignados aleatoriamente a un grupo con levofloxacina o a un grupo placebo). Se utilizó un sistema de aleatorización por bloques permutados de tamaño variable con un enmascaramiento doble basado en sobres sellados, sin posibilidad de conocer la asignación sino hasta el fina del estudio. Después de la aplicación de la levofloxacina o el placebo, se aplicó la cistoscopia y se entregó la orden para la realización del urocultivo para ser tomado entre el 3er y el $10^{\circ}$ día después del procedimiento. Posteriormente en la cita de control se completó el cuestionario con el resultado del urocultivo y la presencia de síntomas urinarios y efectos adversos presentados por el medicamento o complicaciones por el procedimiento. Se realizó un análisis con intención de tratar, se realizó estadística descriptiva, análisis bivariado de las diferentes variables y análisis del riesgo relativo de las variables de resultado final (primario y secundario). Todos los análisis fueron realizados en Stata versión $11 @$. Se cumplieron con los estándares internacionales y nacionales de ética humana. Resultados: Se analizaron 138 pacientes en cada brazo de estudio, después de la exclusión de los pacientes que no cumplieron con el seguimiento ( 9 pacientes). La incidencia de infección urinaria en el grupo intervención fue $0.7 \%$ y en el grupo placebo fue $3 \%(\mathrm{p}=0.17)$ sin encontrar diferencias estadísticamente significativas. La incidencia de bacteriuria asintomática en el grupo intervención fue $5.8 \%$ y en el grupo control fue $14.5 \%(\mathrm{p}=0.01)$ con un NNT=11. Conclusión: No hay diferencias estadísticamente significativas en la utilización de antibiótico profiláctico (Levofloxacino) comparado con no utilizarlo para disminuir la incidencia de infección urinaria en pacientes que son sometidos a cistoscopia como procedimiento ambulatorio con orina estéril demostrada por urocultivo. 
09. Primary school interventions to promote fruit and vegetable consumption: a systematic review and metaanalysis.

Delgado-Noguera $\mathbf{M}^{1}$, Tort $\mathrm{S}^{2}$, Martínez-Zapata $\mathbf{M J}^{3}$, Bonfill $\mathrm{X}^{3}$.

${ }^{1}$ Red Cochrane Iberoamericana, Barcelona, España. Dpto. Pediatría, Universidad del Cauca, Colombia. ${ }^{2}$ Centro Cochrane Iberoamericano, IIB Sant Pau, Barcelona, España.

${ }^{3}$ Centro Cochrane Iberoamericano, IIB Sant Pau, CIBERESP, Universitat Autónoma de Barcelona,

${ }^{3}$ Centro Coct

Background: Daily consumption of $400 \mathrm{~g} /$ day or at least five servings of vegetables and fruits a day, starting from childhood can prevent chronic diseases in the future such as cardiovascular diseases, overweight and obesity. However, epidemiologic data show that the consumption of fruits and vegetables (FV) is insufficient both in adults and children, and their low intake is among the top 10 risk factors contributing to attributable mortality. Schools are a setting for implementing interventions to improve the consumption of FV. Objective: The consumption of FV may contribute to the prevention of many diseases. However, children at school age do not eat an enough amount of those foods. We have systematically reviewed the literature to assess the effectiveness of school interventions for promoting the consumption of FV. Methods: We performed a search in MEDLINE, EMBASE, CINAHL and CENTRAL. We pooled results and stratified the analysis according to type of intervention and study design. Results: Nineteen cluster studies were included. Most studies did not describe randomization method and did not take the cluster's effect into account. Pooled results of two randomized controlled trials (RCTs) of computer-based interventions showed effectiveness in improving consumption of FV [Standardized Mean Difference (SMD) 0.33 (95\% CI 0.16, $0.50)]$. No significant differences were found in pooled analysis of seven RCTs of multicomponent interventions or pooling results of two RCTs evaluating free/subsidized FV interventions. Conclusions: Meta-analysis shows that computer-based interventions were effective in increasing FV consumption. Multicomponent interventions and free/subsidized FV interventions were not effective. Improvements in methodology are needed in future cluster studies. Although these results are preliminary, computer-based interventions could be considered in schools, given that they are effective and cheaper than other alternatives.

\section{Uso de la vacuna antineumocócica conjugada 10-valente y 13-valente en niños previamente vacunados con 7-valente: Revisión sistemática.}

Canelo C, Mezones-Holguín E, Suarez V.

Instituto Nacional de Salud.

Introducción: S. pneumoniae es causa importante de morbi-mortalidad en menores de cinco años. La primera vacuna conjugada pneumococica 7-valente (PCV7) demostró ser eficaz y ha sido ampliamente usada. El año 2011, se informó el retiro de VCP7 del mercado. Dos vacunas conjugadas 10-valente (VCP10) y 13-valente (VCP13) constituyen altrnativas de reemplazo en países en que previamente se usó VCP7. En un grupo de niños se dará el intercambio antes de finalizado el esquema de vacunación. Objetivo: Evaluar la evidencia sobre intercambiabilidad de vacunas en población pediátrica previamente vacunada con VCP7 basado en la no inferioridad inmunológica en el grupo preve a intercanio. Método de Búsqueda: Búsqueda sistemítica en Cochrane Cen Register of Controlled Trials (CENTRAL), MEDLINE, EMBASE y LILACS usando términos pre-especificados. Se requirió información a las empresas farmacéuticas términos pre-especificados. Se requirió información a las empresas farmacéuticas
productoras de VCP. La búsqueda fue realizada hasta Febrero del 2012. Criterio de Selección: ECA que evalúen la respuesta inmunológica (niveles séricos de anticuerpos y/o capacidad funcional opsonofagocitica) comparando un grupo con esquema completo con VCP7 frente a un grupo con intercambio de VCPs antes de finalizado el esquema. Colección de datos y análisis: Tres autores independientemente seleccionaron y extrajeron Coleccion de datos y andisis. Tres autores independientemente seleccionaron y extrajeron Resultados Principales: Dos estudios cumplieron los criterios de inclusion, en ambos estudios tras tres dosis de VCP7 se evaluó el intercambio de la dosis de refuerzo. Un estudio asignó la dosis refuerzo a VCP13 o VCP7 (121 y 133 sujetos). Se observó incremento en los niveles séricos promedio ( 3 a 14 veces) de anticuerpos para los siete serotipos comunes; hubo un menor incremento para los serotipos $9 \mathrm{v}$ y 14 en los que recibieron refuerzo con PCV13. Otro estudio asignó la dosis de refuerzo a VCP10 o VCP7 (238 y 91 sujetos). No hubo aleatorización en la asignación y hubo una pérdida de $\cong 50 \%$ de los sujetos en el grupo de VCP10. En ambos grupos más del $95 \%$ de sujetos fueron respondedores (anticuerpos $\geq 0,20 \mu \mathrm{g} / \mathrm{mL}$ GSK-ELISA) para los serotipos comunes. Conclusión de los autores: Evidencia directa sobre respuesta inmunogénica al intercambio de VCPs es escasa y basada en esquemas de cuatro dosis. Si bien se encontró respuesta inmunológica a los serotipos comunes tras el intercambio en la dosis de refuerzo. El estudio de VCP10 tiene limitaciones metodológicas que podrían limitar su confiabilidad. Es necesaria la realización de estudios que evalúen el intercambio de vacunas en particular cuando no poseen el mismo acarreador.
10. Prevención primaria de Diabetes Mellitus Tipo 2: Sinopsis basada en la evidencia.

Málaga G, Prevost-Ruiz Y, Villena S, Malpartida F, Suarez V, Mezones-Holguín E.

Antecedentes: La Diabetes Mellitus tipo 2 (DM-2) se viene incrementando progresivamente en Perú; por lo que incidir en su prevención primaria se convierte en una necesidad de salud pública. Objetivo: Identificar intervenciones efectivas para la prevención primaria de DM-2 en población prediabética. Métodos: Se llevó a cabo una Sinopsis considerando la información proveniente de estudios de síntesis (EDS): revisiones sistemáticas con meta-análisis, enfocada en intervenciones educativas, higiénico-dietéticas y/o farmacológicas utilizadas para prevenir la DM-2 en población prediabética. Se realizó una búsqueda sin restricción de idioma en el registro de estudios del Grupo COCHRANE y en MEDLINE usando términos pre-especificados, considerando como marco temporal enero del 2001 a abril a del 2011. La búsqueda, selección y evaluación de la calidad de los artículos, fue desarrollada en forma ciega e independiente por dos revisores. Resultados: Se incluyeron 12 publicaciones, las cuales fueron de regular y buena calidad metodológica. Se encontró que la alimentación saludable asociada con actividad física, el consumo de vegetales de hoja verde y la reducción de peso; tuvieron resultado favorable, con una calidad de evidencia sólida. Las intervenciones que incluyen solamente actividad física o la reducción de la porción de alimentos en $10 \%$, fueron favorables; sin embargo, se trata de una evidencia débil. Con relación al aumento en el consumo de frutas, la evidencia no fue favorable. Por otro lado, la evidencia es favorable y sólida para el uso de metformina, pero su recomendación es controversial como medida de salud pública. Conclusiones: Los cambios en el estilo de vida mediante una alimentación saludable asociada con actividad física, el aumento de consumo de vegetales de hoja verde y la reducción de peso cuentan con evidencia sólida para la prevención de DM-2 en personas con prediabetes.

12. Nutrición enteral completa en recién nacidos prematuros menores de 1500 gr al nacer: Comparación de dos esquemas de alimentación en el Hospital Edgardo Rebagliati Martins, 2009.

Cuba L ${ }^{1}$, Hernández $\mathrm{H}^{2}$, Loza $\mathrm{C}^{2}$, Ortiz E $\mathrm{E}^{3}$, Ramírez $\mathrm{A}^{3}$.

${ }^{1}$ Hospital Nacional Guillermo Almenara Irigoyen.

${ }^{2}$ Epidemiólogo Clínico, Universidad Peruana Cayetano Heredia.

${ }^{3}$ Hospital Nacional Edgardo Rebagliati Martin.

Antecedentes: La ganancia de peso de los prematuros menores de 1500 gr al nacer durante la hospitalización, es un problema, incluso usando leche materna con fortificantes comerciales y más aún si no se cuenta con ellos por falta de recursos. En nuestro medio no se conoce los resultados de fortificantes caseros de bajo costo. Objetivos: Comparar la ganancia de peso, talla, perímetro cefálico y variación del z-score con dos esquemas de fortificación de leche materna. Evaluar las variaciones de la hemoglobina, albúmina, calcio, fósforo, fosfatasa alcalina y la estancia hospitalaria con cada esquema de alimentación. Material y métodos: Es un ensayo clínico aleatorizado, controlado a doble ciego. Se estudió 44 recién nacidos con peso menor de 1500 gr. El primer grupo $(\mathrm{n}=22)$, recibió leche materna fortificada Esquema 1 (Casero) y el segundo grupo de iguales características $(\mathrm{n}=22)$ recibió leche materna fortificada Esquema 2 (Comercial). Para comparar variables numéricas sin distribución normal se usó la prueba de rangos de Wilcoxon, y en caso contrario se comparó con el ttest para datos independientes. La variación de la puntuación $\mathrm{Z}$ de peso, longitud y perímetro craneal entre el nacimiento y el alta se evaluaron mediante el ttest para datos apareados. El z-score se calculó utilizando las curvas de crecimiento de Fenton. Resultados: La ganancia de peso ( $\mathrm{gr} /$ día), talla $(\mathrm{cm} / \mathrm{semana})$ y de perímetro cefálico $(\mathrm{cm} / \mathrm{semana})$ fue similar con el Esquema 1 y $2(24,54 \pm 4,45$ vs $25,64 \pm 5,62 ; 0,59 \pm 0,32$ vs $0,54 \pm 0,32 ; 0,62 \pm 0,25$ vs $0,75 \pm 0,21$ respectivamente ( $>0,05$ ), así como su variación del $\mathrm{z}$-score y estancia hospitalaria $(\mathrm{p}>0,05)$. Al finalizar el estudio, los niveles plasmáticos de albúmina, fósforo y delta de calcio fueron menores con el esquema 1 comparado con el Esquema 2 p $<0,05$ ). Conclusiones: Los dos esquemas de fortificación mostraron resultados similares. Los niveles plasmáticos de albúmina, calcio y de fósforo fueron menores con el Esquema 1 , pero dentro de lo normal. 


\section{Adaptación o desarrollo de novo de Guías de Práctica Clínica. Una experiencia en Colombia.}

Gaitán H.

Instituto de Investigaciones Clínicas, Universidad Nacional de Colombia.

Objetivo: Presentar una experiencia colombiana en el proceso de adaptación de guías de práctica Clínica desarrolladas internacionalmente. Metodología: Se hace una revisión de la normatividad vigente que soporta el desarrollo de guías en el contexto colombiano, Se hace una descripción de los problemas encontrados en cuanto a la búsqueda de guías disponibles, la calificación con el instrumento Agree II, el mapeo de la evidencia, el uso de la metodología Grade y la implementación. Resultados: La normatividad Colombiana soporta la inclusión de tecnologías en los planes de aseguramiento con base en el desarrollo de guías de práctica Clínica para patologías específicas, las cuales han sido priorizadas por el Gobierno central. El Ministerio de salud creo una guía metodológica que pretende dirigir al proceso de desarrollo de guías de practica clínica Tres universidades constituyeron una alianza para dar mayor transparencia y garantizar la calidad del proceso. El proceso de desarrollo encontró dificultades para seguir en la práctica la guía propuesta por el Ministerio. El Instrumento AGREE II tiene una limitada concordancia interobservador. El mayor desacuerdo se encuentra entre los expertos clínicos y los metodológicos. El alcance y objetivo de la guías, así como el mapeo de la evidencia y las recomendaciones pueden afectar la decisión de adaptar guías de buena calidad metodológica y conducir más bien al desarrollo de novo de la Guías. El uso de las tablas de resumen de la evidencia propuesto por el grupo GRADE es complejo en su aplicación. La implementación de la guía debe ser tomada en cuenta también el proceso de adaptación. Conclusiones: A pesar de que es aconsejables primero buscar la disponibilidad de guías a nivel internacional, y evaluar su posible adaptación al contexto local, algunas veces es mas adecuado el desarrollo de las mismas.

\section{Revisión de la literatura: tratamiento antituberculoso en pacientes co-infectados con VIH.}

Canelo C, Donaires F, Solari L.

Instituto Nacional de Salud

Introducción: El 2007, se reportó que 1,3 millones (14,8\%) estaban coinfectados con $\mathrm{TBC} / \mathrm{VIH}$. Las personas con VIH/TBC tienen mayor riesgo de progresar a enfermedad activa y mortalidad. Regímenes de corta duración han mostrado ser eficaces en tratar TBC en personas inmunocompetentes. La duración óptima de la terapia para personas con en personas inmunocompetentes. La duración optima de la terapia para personas con
coinfección es controversial. Objetivos: Evaluar la evidencia sobre la eficacia de terapia
antituberculosa extendida ( $\geq$ ocho meses) frente a esquema de corta duración (seis meses) antituberculosa extendida ( $\geq$ ocho meses) frente a esquema de corta duración (seis meses)
en pacientes coinfectados con VIH sobre: mortalidad, falla al tratamiento y recurrencia de enfermedad. Método de Búsqueda: Búsqueda sistemática en Cochrane Central Register of Controlled Trials (CENTRAL), OVID MEDLINE, EMBASE y LILACS usando términos pre-especificados hasta febrero del 2012. Criterio de Selección: ECA de tratamiento de tuberculosis en individuos con infección VIH. Diagnóstico clínico o bacteriológico de tuberculosis no tratado previamente, comparando dos brazos de tratamiento de diferente tuberculosis no tratado previamente, comparando dos brazos de tratamiento de diferente
duración. El brazo de menor duración debía durar $\geq 6$ meses. Colección de Datos $y$ duración. El brazo de menor duración debía durar $\geq 6$ meses. Colección de Datos y
Análisis: Dos autores independientemente seleccionaron y extrajeron datos. Dos revisores independientemente evaluaron la calidad metodológica. Resultados Principales: Cuatro estudios cumplieron los criterios de inclusión, todos fueron de etiqueta abierta. En dos de ellos no se describió el método de generación de aleatorización o el ocultamiento de códigos. La mortalidad no fue evaluada homogéneamente, unos reportaron mortalidad durante tratamiento y otros posteriores al fin de terapia. No se incluyó mortalidad durante terapia por no ajustar los diferentes tiempos en riesgo. Un estudio reportó a los 36 meses terapia por no ajustar los diferentes tiempos en riesgo. Un estudio reporto a los 36 meses
del reclutamiento $17,3 \%$ de mortalidad en el grupo de 6 meses y $20,5 \%$ en el grupo de 9 del reclutamiento $17,3 \%$ de mortalidad en el grupo de 6 meses y $20,5 \%$ en el grupo de 9
meses. Otro estudio con 24,2 meses de seguimiento promedio reportó 27,1 muertos por meses. Otro estudio con 24,2 meses de seguimiento promedio reportó 27,1 muertos po
100 personas-años y 21,1 muertos por 100 personas-año en el grupo de 9 y 6 meses respectivamente. Ambos resultados no estadísticamente significativos. Un meta-análisis de efectos aleatorios no mostró diferencias en la tasa de falla terapéutica, pero si un reducción en el ieso de ECA es escaso en relación a al impacto de la condición. No se encontró diferencias en mortalidad por duración de esquema. Los datos reportados muestran menor tasa de recaída, sin embargo estos no diferenciaron entre reactivación y reinfección lo que limita su interpretación. Estudios adicionales se requieren para determinar fehacientemente el efecto de la terapia extendida.

16. Evaluación del Test Diagnóstico Índice Número de Plaquetas/ Diámetro Mayor del Bazo, como Predictor de la Presencia de Varices Esofágicas en Cirrosis Hepática. adversos en la Clínica Carlos Lleras de Bogotá, Colombia 2009-2010.

Ovalle D, Gaitán H, Arteaga JM, Granados C, Eslava J, Guevara O.

Evaluación de Políticas y Tecnología en salud y Equidad en salud, Facultad de Medicina, Universidad Nacional de Colombia.

Introducción: Los eventos adversos intrahospitalarios son un problema de salud pública a nivel global. Poco se conoce de sus consecuencias en costos para los sistemas de salud en Latinoamérica. Objetivo: Determinar la Frecuencia y costos de los Eventos Adversos (EA) así como los determinantes asociados, que afectan a los pacientes en los servicios quirúrgicos y médicos en e la Clínica Carlos Lleras. Materiales y métodos: Cohorte prospectiva. Pacientes hospitalizados en los servicios de cirugía (Qx), medicina interna (MI) en el 2010. Criterios de exclusión: Alteraciones psiquiátricas, evento adverso originado en otra institución y menores de 16 años. Muestra: 632 sujetos en QX y 1265 en MI. Estudio aprobado por el comité de Ética de la institución. Procedimiento: 1. Fase de tamización y fase de calificación del EA. Variables a medir: características socio demográficas, comorbilidad previa, eventos de detección, evento adverso, evitabilidad, costos directos. Identificación de los recursos asociados con cada uno de los eventos identificados como parte del estudio principal para tener el informe del costo total de los Eventos Adversos para Colombia y para cada paciente. Resultados: Ingresaron $397(33,08 \%)$ sujetos a QX y $803(66,92 \%)$ a MI. Prevalencia de eventos dedetección: 10,1\% (IC95\% 8,38-11,79. Incidencia de EA en Qx, 7,3\% (IC 95\%4,73- 9,88) en MI 5,1\% (IC 95\% 3,58-6,63). El $68 \%$ de estos eran evitables. Los factores asociados al EA fueron la comorbilidad previa OR 1,34 (IC 95\%:1,09-1,66) y procedimientos invasivos con un OR: 2,01 (IC 95\%: 1,50-2,71). La media de costos por paciente fue de \$ $1.148 .618,79$. Costo total de los eventos adversos para el sistema de salud colombiano \$55.713.481.199. Conclusión: Los EA afectan una importante población hospitalizada e la Clínica Carlos Lleras y tienen importante repercusión en los costos.
Rodríguez-Hurtado $\mathrm{D}^{1}$, Mosqueira $\mathrm{JR}^{2}$, Montiel $\mathrm{JB}^{2}$, Monge $\mathrm{E}^{3}$.

Médico Internista - Geriatra del Hospital Nacional Arzobispo Loayza. Miembro de la Red Internacional de Epidemiología Clínica (INCLEN).

Médico Cirujano.

Médico Gastroenterólogo del Hospital Daniel Alcides Carrión, Lima-Perú.

Objetivo: Evaluar el índice número de plaquetas/diámetro mayor del bazo (P/DMB) como un método diagnóstico no invasivo de la presencia de várices esofágicas (VE) en pacientes con cirrosis hepática. Material y métodos: Estudio de Test Diagnóstico, análisis retrospectivo. Se recolectaron los datos de pacientes con el diagnóstico de cirrosis hepática, en el Servicio de Gastroenterología del Hospital Nacional Daniel Alcides Carrión (HNDAC), entre Septiembre 2010 y Enero 2011; posteriormente se calculó la sensibilidad, especificidad, valor predictivo positivo y negativo, razón de probabilidades positiva y negativa; utilizando a la endoscopía digestiva alta como patrón de oro. Resultados: Se evaluaron los datos de 47 pacientes, la edad promedio fue de 60,74 años, la etiología fue consumo de alcohol en el $25,5 \%$ y no y 74,5\% las presentaron. El índice $\mathrm{P} / \mathrm{DMB}$ se comparó con la endoscopía, encontrándose una Sensibilidad de $40 \%$, Especificidad de $75 \%$, Valores predictivos positivo y negativo de $82 \%$ y $30 \%$ respectivamente, y razón de probabilidades positiva y negativa de 1,6 y 0,8 respectivamente. Conclusiones: El índice P/DMB no es una prueba diagnóstica efectiva para el diagnóstico de la presencia de VE en la muestra estudiada. determinada en el $48,9 \%$ de pacientes. El $25 \%$ de pacientes no presentaron VE 


\section{Eficacia y seguridad del uso de un sistema uterino liberador de Levonorgestrel (SIU-LNG) para el tratamiento de la menorragia idiopática.}

Villamil E, Aleman A, Pérez A.

División Tecnologías Sanitarias, Ministerio de Salud Pública, Uruguay.

Introducción: El sangrado menstrual cíclico excesivo o menorragia puede ocurrir solo o en combinación con otros síntomas y no está asociado con mortalidad significativa. Un $10 \%$ de mujeres entre los 30 y 55 años sufre pérdida de sangre suficientemente severa para causar anemia y ser clínicamente diagnosticada como menorragia idiopática. El tratamiento de esta patología incluye el tratamiento hormonal, la ablación endometrial y la histerectomía. Objetivo: Evaluar la eficacia y seguridad de un Sistema uterino liberador de levonorgestrel (SIU-LNG) en el tratamiento de la menorragia idiopática. Método: La metodología empleada fue la búsqueda sistemática de ensayos clínicos aleatorizados, revisiones sistemáticas y meta-análisis en Medline/Pubmed, Lilacs y la Colaboración Cochrane. La evidencia encontrada fue evaluada críticamente y se realizó la síntesis de los resultados de la evidencia realizando meta análisis cuando fuera posible. Resultados: El Sistema uterino liberador de levonorgestrel (SIU-LNG) mostró ser significativamente más efectivo que la noretisterona (días 5 a 26 del ciclo menstrual), aunque ambas tecnologías mostraron eficacia en la reducción del sangrado menstrual. La eficacia en la reducción del sangrado fue mayor en la ablación endometrial y la histerectomía pero los efectos adversos fueron menores y menos graves con el uso del SIU-LNG. La tasa de satisfacción y los cambios en la calidad de vida en la ablación endometrial y con uso del SIULNG fueron similares. Respecto s los costos, el SIU-LNG estuvo asociado a menores costos que la histerectomía, al año y a los 5 años de seguimientos de las pacientes. Conclusiones: La evidencia obtenida ubica al SIU-LNG como una alternativa terapéutica aceptable para el tratamiento de la metrorragia idiopática.

19. Revisión sistemática y evaluación de calidad de guías de práctica clínica para el manejo del síndrome coronario agudo.

Acosta N.

Universidad de Antioquia; Medellín, Colombi

Antecedentes: A pesar de los efectos causados por la enfermedad aterosclerótica y en especial de su manifestación como síndrome coronario agudo (SCA), existe evidencia suficiente para demostrar que una intervención adecuada respaldada en una Guía de Práctica Clínica (GPC), permite modificar la progresión de la enfermedad y minimizar el daño, con la consecuente disminución en la mortalidad y la mejoría en la calidad de vida. Por lo tanto, en el marco de la elaboración de la GPC en Colombia, para la detección temprana, atención integral, seguimiento y rehabilitación de pacientes con SCA, se realizó una revisión sistemática y análisis de la calidad de las GPC publicadas para esta condición. Métodos: Mediante búsqueda sistemática, manual y recomendación de expertos, se identificaron GPC para SCA con y sin elevación del ST, publicadas desde el año 2005 hasta el 2011. Cada guía fue calificada con el instrumento AGREE II por dos evaluadores independientes. Cuando hubo discrepancia entre los dos evaluadores en al menos un ítem, mayor o igual a 4 puntos, la guía fue recalificada. Resultados: 121 GPC fueron identificadas. 28 guías cumplieron los criterios de inclusión y se les evaluó su calidad. El promedio total para las 28 guías en los todos los dominios fue: Alcance y Objetivos $78 \%$, participación de los implicados $62 \%$, bajo rigor metodológico $72 \%$, claridad de la presentación $91 \%$, aplicabilidad $44 \%$, independencia editorial $72 \%$. 11 guías evaluadas tenían un bajo rigor metodológico (puntaje menor del 60\%). Conclusiones: Tener en cuenta los puntos de vista de la población diana y la determinación de herramientas para implementar las recomendaciones de la guía, independiente del rigor metodológico; pueden ser los puntos a fortalecer en una GPC, para mejorar el impacto en la morbimortalidad de los pacientes con SCA.
18. Clinical practice guidelines for acute diarrhea in children: methodological quality.

Flórez ID ${ }^{1}$, Lozano $\mathrm{JM}^{4}$, Sierra $\mathrm{JM}^{1}$, Granados $\mathrm{C}^{2}$, Contreras Javier, Tamayo $\mathrm{ME}^{1}$, Briceño $\mathbf{G}^{3}, \mathbf{L u g o ~}_{\mathrm{LH}^{1}}$, Acosta $\mathrm{JL}^{5}$

Universidad de Antioquia; Medellin, Colombi

Pontificia Universidad Javeriana; Bogotá, Colombia

Fundación Cardio-infantil; Bogotá, Colombia.

University of Florida, Miami, USA.

Universidad del Norte, Barranquilla, Colombia.

Background: Before the development of a clinical practice guideline (CPG) it is advisable to search and to assess published guidelines in order to consider its adaptation. Acute diarrhea in children is associated with high mortality in undeveloped countries and high morbidity in developed ones. There are published guidelines of management of diarrhea in children and its quality hasn't been assessed. Objective: To conduct a systematic review of the literature of acute diarrhea or gastroenteritis in children CPG and to assess its quality with the AGREE-II instrument. Methods: We searched electronic databases, national clearinghouses and we also did a search of non electronic sources, until august 2011. Each guideline was assessed with the spanish version of AGREE-II by three clinical epidemiologists. Results: We found 63 guidelines, $17(26.9 \%)$ were evidence-based. Based on AGREE-II, domains 1 and 4 had the highest scores, while domains 5 and 6 , the lowest. Six guidelines scored over $60 \%$ in the third domain and they were considered recommended $\mathrm{CPG}$, and 11 were considered non-recommended guidelines. The recommended CPG had low scores in the domains 5 and 6 , and high scores in the domains 1 and 4, as non-recommended ones. Discussion: It is advisable to improve the quality of GPC of diarrhea. Most of the guidelines were not evidence-based. CPG developers are concerned in writing down correctly objective, scope and population of the guideline but they are not concerned about methodological quality. Implications: The forth domain should be the one that discriminate the quality of $\mathrm{CPG}$.

\section{Uso de modelos epidemiológicos para estimar la incidencia de caries dental y enfermedad periodontal en embarazadas chilenas.}

Corsini-Muñoz G, Zaror-Sánchez C, Vallejos-Vallejos C.

Centro de Excelencia CIGES, Universidad de La Frontera; Temuco, Chile.

Antecedentes: Existe la necesidad de contar con datos epidemiológicos de incidencia de caries y enfermedad periodontal en embarazadas, los cuales son de gran utilidad en la planificación de los recursos sanitarios, en la toma de decisiones sobre nuevas intervenciones, y en la evaluación de la eficiencia de estas. Objetivos: Determinar la incidencia de caries y enfermedad periodontal en embarazadas chilenas a partir de los datos disponibles de prevalencia y mortalidad, usando el programa epidemiológico DisMod II. Materiales y métodos: Se construyó un modelo teórico de cada enfermedad en DisMod II, basándose en los datos de prevalencia estimada en la Encuesta Nacional de Salud de año 2003 en Chile, y en los datos del Estudio de Carga de Enfermedad en Chile del año 2007. Para efectos de estimar la población de mujeres embarazadas, se utilizó como variable de aproximación, los partos por edad de las mujeres según la información de los nacimientos registrado por el Instituto de Nacional de Estadística de Chile (INE). Los datos fueron ingresados en el programa DisMod para determinar las incidencias anuales por grupos etarios, y comprobar la consistencia interna del modelo. Resultados: La incidencia de caries estimada por el modelo fue de 86.870 nuevos casos de caries en el subgrupo de embarazadas, con una duración promedio de la enfermedad de 1,9 años, y en la enfermedad periodontal fue de 7983 casos incidentes al año, con una duración promedio de la enfermedad de 3,2 años, en un universo estimado de 230.831 mujeres embarazadas chilenas. Conclusiones: Los modelos epidemiológicos son una herramienta útil para determinar la epidemiología de cualquier enfermedad, especialmente en aquellos casos en que no existen datos de incidencia, y cuyo estudio en terreno sería muy costoso y de larga duración. 


\section{Revisión sistemática sobre la terapia de reposición de fluidos en pacientes con dengue según gravedad.}

Donaires F, Fiestas V, Pachas P, Zavala R, López J, Suarez V. Instituto Nacional de Salud

Antecedentes: El dengue es la arbovirosis más importante que produce enfermedad en el humano. Actualmente no se dispone de tratamiento específico de la infección por dengue, por lo que se debe seleccionar dentro de lo disponible, el mejor abordaje terapéutico de los casos, ante la inminente expansión del DEN-2 genotipo América/Asia, que vienen condicionando la aparición de formas graves. Objetivo: Evaluar la terapia de reposición de fluidos más eficaz en el manejo de pacientes niños y adultos con dengue. Material y métodos: Se realizó la búsqueda en las bases de datos Medline, Cochrane BVS y Lilacs, fueron incluidos los estudios indexados al 15 de marzo del 2011, que evaluaron la hidratación con cristaloides y coloides en el manejo de dengue. La calidad de los estudios fueron evaluados con el score CASP: Cohort Studies, para los estudios de cohorte, y las guías de evaluación crítica de estudios de terapias publicados en serie JAMA, para los ensayos clínicos. Resultados: Se identificaron 140 artículos relevantes: 126 en Medline, 11 en Cochrane BVS y tres en Lilacs; de las cuales se seleccionaron siete publicaciones. En el Dengue Hemorrágico (DH) con shock y Síndrome de shock por Dengue (SSD), se identificaron tres ensayos clínicos realizados en niños que no encontraron diferencia significativa con respecto a la ocurrencia de nuevos episodios de shock, volumen total de fluidos, recuperación final, días de hospitalización y frecuencia de complicaciones entre el uso de coloides y cristaloides. Un cuarto ensayo clínico, no encontró diferencia significativa entre los dos coloides evaluados. Un estudio cuasiexperimental que comparó lactato de Ringer con Haes-Steril 6\%, tampoco encontró diferencia significativa de la presión oncótica, recurrencia del shock, estancia hospitalaria en UCI, y la media del hematocrito antes y después del bolo inicial de fluido. En lo que respecta al dengue clásico y $\mathrm{DH}$ sin shock se identificó un estudio descriptivo realizado en niños y adultos, que mostró beneficio de la hidratación oral en disminuir la tasa de hospitalización y la severidad del dengue. Conclusiones: Los ensayos clínicos desarrollados en población pediátrica no encontraron diferencias en la recuperación del estado de hidratación con el uso de coloides versus cristaloides en los pacientes con DH sin shock y SSD. No existen ensayos clínicos en las bases bibliográficas revisadas que evalúen la terapia de reposición de fluidos en dengue clásico y DH sin shock en adultos y niños.

\section{Odontología basada en la evidencia: Importancia clínica del índice de masa corporal en la incidencia de la enfermedad periodontal.}

Monsalves MJ, Raposo A, Lara A, Hofer P.

Universidad de La Frontera; Temuco, Chile.

Antecedentes: Muchos estudios epidemiológicos señalan una asociación entre la obesidad y la enfermedad periodontal. Sin embargo, la tendencia actual va más allá y pretende estudiar la asociación entre el índice de masa corporal (IMC) y la incidencia de la Enfermedad Periodontal (EP). Objetivo: Conocer la asociación entre el IMC y la incidencia de la EP. Material y métodos: Se realizó una revisión de los estudios que asociación el IMC y la incidencia de enfermedad periodontal en los últimos 5 años. Para esto se revisaron las bases de datos de Medline, Scielo y revistas especializadas en periodoncia o salud oral. Los artículos se revisaron por 3 investigadores independientes para evaluar calidad de las investigaciones, para esto se utilizaron guías de revisión de estudios según su tipo de diseño. Resultados: Se encontraron un total de 199 artículos relacionados, por lo que la búsqueda se acotó a estudios de mayor evidencia, revisiones sistemáticas y ensayos clínicos, obteniendo un número final de 19 estudios. Conclusiones: La mayoría de los estudios presenta una significativa asociación entre el IMC y la enfermedad periodontal, principalmente relacionado a la presencia de patologías como la Diabetes Mellitus, mal control metabólico y factores de riesgo como el tabaco, en el grupo de pacientes obesos. La mayor parte de los artículos señala como indicador de riesgo el sobrepeso y la obesidad en la incidencia de Enfermedad Periodontal.

\section{Suplementación de hierro a niños en zonas endémicas de malaria: Sinopsis basada en la Evidencia.}

Mezones-Holguín E, Gutiérrez-Aguado A, Hijar G, Suarez V. Instituto Nacional de Salud.

Antecedentes: La anemia por deficiencia de hierro es frecuente en la infancia en países en vías de desarrollo; siendo la suplementación oral con hierro, una medida utilizada para contrarrestarla. Sin embargo, algunos países han suspendido esta intervención en zonas endémicas de malaria. Objetivos: Identificar, revisar y evaluar la mejor evidencia disponible acerca del uso de suplementación oral con hierro a niños en zonas endémicas de malaria. Métodos: Se llevó a cabo una Sinopsis considerando la información provista por Estudios de Síntesis (EDS): revisiones sistemáticas (RS). Se realizó una búsqueda sin restricción de idioma en TRIPDATABASE, COCHRANE, MEDLINE, LILACS y SCIELO usando términos pre-especificados, considerando investigaciones publicadas hasta octubre del 2011. La búsqueda y selección de los artículos, así como la evaluación de calidad, fue desarrollada en forma ciega e independiente por dos revisores. Resultados: Se encontró una RS Cochrane y su respectiva actualización, la cual incluía todos los ensayos clínicos aleatorizados publicados hasta junio del 2011. En este artículo se consideraba como desenlaces primarios la malaria sintomática, malaria severa y muerte. Esta tuvo también un análisis por subgrupo considerando la presencia de anemia previa a la suplementación, la edad y el grado de endemicidad de la zona. Este EDS no encuentra asociación entre la suplementación y un incremento en los desenlaces primarios elegidos tanto a nivel general como por subgrupos. Esta revisión tuvo un bajo riesgo de sesgos tratándose de una evidencia sólida. Sin embargo, no se ha realizado un sub-análisis de acuerdo a la especie de plasmodium. Conclusiones: Existe evidencia sólida de que la suplementación oral con hierro en niños no conduce a un incremento en la incidencia de malaria sintomática, malaria severa y muerte en zonas endémicas de malaria.

\section{Malnutrición fetal como factor pronóstico de morbilidad neonatal precoz.}

Salazar-Alarcón JL ${ }^{1}$, Núñez-Guevara $\mathbf{J C}^{1}$, Sosa-Flores $\mathbf{J L}^{1,2}$, Díaz-Vélez $\mathbf{C}^{\mathbf{1}, 2}$.
Universidad Nacional Pedro Ruíz Gallo; Chiclayo, Perú.

Universidad Nacional Pedro Ruíz Gallo; Chiclayo, Perú.

Hospital Nacional Almanzor Aguinaga Asenjo; Chiclayo, Perú.

Antecedentes: Los efectos del mal estado nutricional en los primeros años de vida se prolongan a lo largo de toda la vida, incrementando el riesgo de padecer enfermedades crónicas. Adebami y col. concluyeron que la asfixia perinatal, dificultad respiratoria, aspiración de meconio, hipoglucemia, hematocrito elevado e hipoalbuminemia son más frecuente en recién nacidos (RN) con malnutrición fetal; además tiene mortalidad y secuelas neurológicas significativamente mayores en el periodo neonatal. Objetivo: determinar el riesgo de morbilidad neonatal precoz en recién nacidos a término por parto de vaginal que presentan malnutrición fetal. Materiales $y$ métodos: estudio analítico prospectivo tipo cohorte dinámica. Población: recién nacidos a término sanos (RNTS) nacidos por parto vaginal del hospital regional docente Las Mercedes. Muestra: calculada con el programa EPIDAT versión 3.1 considerándose una razón de no expuestos sobre expuestos para sospecha de sepsis neonatal de $20 \%$ en recién nacidos con malnutrición fetal, nivel de confianza $95 \%$ y potencia de $70 \%$, la muestra fue de 49 recién nacidos a término con malnutrición fetal y 245 recién nacidos sin malnutrición fetal. Resultados: $46(15,6 \%)$ RNTS con malnutrición fetal y $248(84,4 \%)$ sin malnutrición fetal, los recién nacidos expuestos a malnutrición fetal presentaron 4,18 y 3,34 veces mayor riesgo para desarrollar morbilidad y policitemia que aquellos sin malnutrición fetal respectivamente $(p<0,05)$, al inicio del séptimo día la probabilidad de sobrevida libre de morbilidad, en RN sin malnutrición fetal es 0,78 [IC 95\% 0,73-0,83] comparado con solo un 0,25 [IC 95\% 0,13-0,41] de aquellos con malnutrición (Log Rank <0,05). Conclusión: Los RNTS con malnutrición fetal presentan riesgo para desarrollar morbilidad y policitemia, además la sobrevida libre de morbilidad es mayor en RNTS sin malnutrición fetal durante el periodo neonatal precoz. 


\section{Efectividad de la suplementación preventiva con zinc para disminuir la incidencia, duración y gravedad de los episodios de diarrea en niños menores de 5 años.}

Sierra JM, Flórez ID, Contreras JO, Cornejo JW, Bustamante A. Grupo de Investigación Clínica en Enfermedades del Niño y del Adolescente, Universidad de Antioquia; Medellín, Colombia.

Antecedentes: La prevalencia de deficiencia de zinc en niños en Latinoamérica es alta; existen revisiones sistemáticas sugieren que su suplementación de zinc además de ser útil en tratamiento, disminuye la incidencia de diarrea, aunque con alta heterogeneidad en resultados: estas incluían estudios cuyo desenlace primario no era incidencia de diarrea. Objetivo: Determinar la efectividad de la suplementación preventiva con zinc en ensayos clínicos para disminuir la incidencia de diarrea, la duración de los episodios en menores de 5 años. Materiales y métodos: Revisión sistemática de la literatura y metanálisis. Se revisó MEDLINE, EMBASE, LILACS, CENTRAL y literatura gris. Se incluyeron ensayos clínicos aleatorizados de efectividad y seguridad de zinc vs placebo en niños menores de cinco años que incluyeron como desenlace principal la incidencia de diarrea. Revisiones de estudios por dos evaluadores independientes. La calidad se evaluó según desenlace con sistema GRADE. Análisis de subgrupos según sal utilizada, forma de dosificación, y duración de suplementación. Se evaluó la heterogeneidad antes de combinar resultados. Resultados: Se incluyeron 8 estudios. El RR para incidencia de diarrea fue 0,90 (IC95\% 0,85-0,95). Los resultados tenían moderada heterogeneidad $\left(\mathrm{I}^{2}=59 \%\right)$. Por subgrupos la suplementación diaria fue efectiva sin heterogeneidad ( $\mathrm{RR} 0,88$; IC95\% $0,84-0,92, \mathrm{I}^{2}=0 \%$ ). ) a diferencia de suplementación semanal (RR 0,$92 ; \quad$ IC95\% $\quad 0,81-1,03 \quad \mathrm{I}^{2}=79 \%$ ). La suplementación mayor de tres meses y la de menos de tres, fueron efectivas. Gluconato, acetato y sulfato de zinc fueron efectivos si se administraban diariamente. La calidad de la evidencia fue moderada. Discusión: La suplementación diaria de zinc es efectiva para disminuir la incidencia de diarrea en niños menores de cinco años, no así la suplementación semanal. Todas las sales son efectivas. La inclusión solo de estudios que tuvieron en cuenta nuestro desenlace como desenlace primario disminuyó la heterogeneidad al compararse con otros metanálisis en el tema.

\section{Experiencia en la elaboración de evaluaciones económicas en las Guías de Atención Integral de Colombia.}

Mejía A, Atehortúa S.

Universidad de Antioquia; Medellín, Colombia.

Antecedentes: Ineficiencias en la prestación de servicios, el incremento en recobros por medicamentos y procedimientos no incluidos en el plan de beneficios y los altos costos en el Sistema General de Seguridad Social en Salud (SGSSS) en Colombia, amenazan su sostenibilidad financiera, disminuyen la calidad en la atención y profundizan las inequidades en salud. Estas consideraciones motivaron la realización de Guías de Atención Integral (GAI) basadas en la evidencia, las cuales incluyeron un componente de evaluación económica. Objetivos: Evaluar el proceso de priorización y diseño de las evaluaciones económicas incluidas en las GAI colombianas. Metodología: Evaluación metodológica del proceso de selección de preguntas y recomendaciones prioritarias para evaluación económica, y de la definición y conducción de las evaluaciones económicas de novo incluidas en las GAI. Se identificaron las principales dificultades en cada uno de los pasos planteados en la Guía Metodológica para la elaboración de GAI en el SGSSS Colombiano y se ofrecen recomendaciones para mejorar el proceso. Resultados: 1) La selección de preguntas para las cuales se realizará evaluación económica no debería definirse antes de la búsqueda de evidencia. Se sugiere identificar las preguntas que podrían ser prioritarias y realizar la búsqueda de evidencia para estas; una vez se tengan los resultados, se define a cuál recomendación realizar el estudio económico. 2) La información sobre costos es muy heterogénea y fragmentaria. Se sugiere al Ministerio de Salud elaborar un manual de costos de referencia para evaluaciones económicas, que permita homogenizar el costeo y facilite el uso de los resultados para la toma de decisiones. 3) No es factible realizar estudios de novo para todas las recomendaciones clasificadas como prioritarias. Sugerimos incluirlos en la agenda de investigación del Gobierno y Universidades. 4) Es clave fomentar la formación en evaluación económica a nivel gubernamental y en los programas de epidemiología clínica.
26. Intervenciones dirigidas a la prevención de eventos adversos basadas en sistemas de gestión de riesgo clínico en instituciones hospitalarias. Una revisión sistemática.

Torres $\mathbf{A}^{\mathbf{1}}$, Gaitán $\mathbf{H}^{\mathbf{1}}$, Reveiz $\mathbf{L}^{2}$.

Grupo de Evaluación de Tecnologías y Políticas en Salud, Universidad Nacional de Colombir.

Organización Panamericana de la Salud.

Introducción: La seguridad de los pacientes se ha convertido en una de las principales prioridades a nivel mundial, llevando a la generación de políticas dirigidas a incentivar la implementación de sistemas de gestión de riesgo clínico (SGRC) para la prevención de eventos adversos prevenibles. Objetivo: Determinar los componentes y la efectividad de los diferentes sistemas de gestión de riesgo para la prevención de eventos adversos hospitalarios, en el marco de la evaluación de tecnologías. Métodos: Se realizó una revisión sistemática mixta con un componente cuantitativo que identifica desenlaces de efectividad (Ej. eventos adversos prevenidos, estancia hospitalaria, índice de Criticalidad) y un componente cualitativo que identifica los pasos y características de cada SGRC y cómo funcionan en sus contextos particulares. Se utilizó la metodología del Grupo Cochrane de Salud pública y la del Centro Eppi con integración de los dos componentes en los resultados. Resultados: Se incluyeron 28 estudios para el componente cuantitativo de efectividad y 125 estudios que identifican la operativización de los SGRC. Se identificaron 7 SGRC reportados en la literatura. Existe evidencia de baja calidad y para alguno SGRC evidencia contradictoria para medir la efectividad de 4 SGRC. Se crearon tablas comparativas como producto de una meta-síntesis de información con el fin de comparar los SGRC. Discusión: No existe evidencia de alta calidad suficiente para identificar cual es el SGRC más efectivo para la prevención de eventos adversos. Sin embargo, existen varios SGRC con metodologías sistemáticas, reproducibles que permiten ser utilizadas en diferentes contextos y en cualquier tipo de proceso hospitalario como Análisis de Modo de Falla y Efectos y Métodos de Mejoramiento Continuo de la Calidad. Se necesita mayor investigación con mayor rigor metodológico y un mayor avance en las metodologías para intervenciones compuestas.

\section{Prácticas clínicas basadas en la evidencia en atención perinatal: experiencia del de un servicio materno infantil en Uruguay.}

Aleman A, Prina S, Papazzian M, Rua F, Servetto M.

Gerencia de Salud, Banco de Previsión Social, Uruguay.

Antecedentes: El uso de prácticas clínica de efectividad probada por evidencia científica de alta calidad es una de las estrategias para mejorar la calidad de atención y disminuir la variabilidad de la práctica clínica. En el control prenatal y la atención del parto existen prácticas de probada efectividad que no siempre son utilizadas con alta frecuencia en los servicios de salud. Objetivos: Determinar la frecuencia de realización de prácticas clínicas basadas en evidencias en el control prenatal y la atención del parto en el servicio materno infantil del Banco de Previsión Social del Uruguay. Métodos: Se identificaron las prácticas clínicas basadas en la evidencia que se registran en el sistema informático perinatal. Se realizaron los análisis de frecuencias de las variables identificadas en forma aislada y luego sumatoria para cada registro del año 2011. Las prácticas incluidas fueron: administración de ácido fólico, vacuna antitetánica vigente, corticoides en parto pretérmino, inmunoglobulina antiD en mujeres RH negativas, episiotomía selectiva, manejo activo del parto, cesárea en podálica. Resultados: Se analizaron 1727 registros El análisis preliminar mostró que la frecuencia de administración de folatos fue de $53 \%$, la episiotomía en mujeres primíparas es $52 \%$, el manejo activo del alumbramiento fue del $58 \%$ y la cesárea en presentación podálica 97\%. Discusión: Los resultados muestran una frecuencia baja de uso de prácticas basadas en la evidencia aun cuando la mayoría de las mismas son recomendadas por las guías nacionales. Conclusiones: Es necesario establecer mecanismos para promover la disminución de la variabilidad en la práctica clínica y promover el uso de guías de práctica basadas en la evidencia. 


\section{Identificación de ensayos clínicos en publicaciones periódicas en Uruguay 2000-2010.}

\author{
Gianneo $\mathrm{O}^{1}$, Fernández $\mathrm{A}^{2}$, Patrón $\mathrm{C}^{3}$, Forster $\mathrm{T}^{4}$. \\ Fondo Nacional de Recursos. \\ Organización Panamericana de la Salud. \\ Facultad de Odontología - Universidad de la República
}

Centro de Documentación e Información Científica, Uruguay.

Antecedentes: Este trabajo es realizado en el marco del proyecto de identificación de ensayos clínicos controlados de la Red Cochrane Iberoamericana. Un primer análisis fue realizado en el año 2010. Objetivos: 1. Identificar ensayos clínicos controlados según la definición de la Colaboración Cochrane Iberoamericana publicados en las revistas biomédicas uruguayas en el periodo 2000-2010. 2 Clasificar los ensayos clínicos detectados según los criterios adoptados en la Colaboración Cochrane Iberoamericana. 3. Identificar Revisiones Sistemáticas con metanálisis publicadas en el período. Material y métodos: Se revisaron las publicaciones periódicas biomédicas publicadas en Uruguay desde enero de 2000 hasta diciembre de 2010, corrientes al inicio del periodo, que cumplieran con las normas internacionales de presentación de publicaciones periódicas, cuyo contenido principal fuera estudios de tipo epidemiológico. Las revistas seleccionadas fueron analizadas en su totalidad según los criterios definidos en el protocolo "Identificación y descripción de ensayos clínicos controlados: Guía de búsqueda manual" de la Colaboración Cochrane Iberoamericana. Los artículos fueron clasificados como ensayos clínicos randomizados, ensayos clínicos controlados y meta-análisis. Resultados: Fueron seleccionadas 13 publicaciones periódicas y se revisaron 1690 artículos. En el relevamiento se identificaron en primera instancia 42 ensayos clínicos sobre los cuales se aplicaron los criterios de evaluación establecidos en el protocolo descartándose 17 artículos. Las razones de exclusión fueron: estudio experimental en animales, piezas odontológicas extraídas, pruebas de laboratorio, entre otros. De los 23 artículos seleccionados 11 son ensayos controlados con distribución aleatoria (RCT), 12 ensayos clínicos controlados (CCT). Se identificaron además 2 metanálisis. Conclusiones: En una búsqueda realizada en PubMed para el mismo periodo, estableciendo Uruguay como país de afiliación de los autores y ensayos clínicos y meta-análisis como tipo de estudio, se recuperaron 71 artículos. Este resultado podría explicar la baja tasa de publicación en revistas uruguayas, indicando la necesidad de un estudio más profundo de la distribución de estos artículos en el periodo considerado. Al mismo tiempo estaría indicando que los científicos uruguayos prefieren publicar este tipo de estudio en revistas extranjeras prestigiosas o en grupos internacionales de trabajo que les brinda una mayor visibilidad.

\section{Validación de Kidscreen-27 versión padres Colombia.}

Vélez CM, García HI.

Universidad de Antioquia; Medellín, Colombia.

Antecedentes: La investigación en Calidad de Vida (CV) en niños es un campo que viene ganado importancia. En Colombia el único instrumento validado es KIDSCREEN, el cual está conceptualmente basado en la definición de CV como un constructo multidimensional. De las tres versiones existentes, sólo se han validado completamente la de 52 ítems y la 27 en su versión para niños. Objetivos: Validar la versión KIDSCREEN-27 para padres, de tal manera que el instrumento completo quede disponible para utilizarse en el contexto colombiano. Material y métodos: Estudio observacional de validación de una escala. La población de estudio fueron padres de niños entre los 8 y 18 años de Medellín, Colombia. Se calculó una muestra de 1.150 padres de acuerdo a las diferentes propiedades psicométricas a medir. Se realizó una validación de constructo comparando las puntuaciones medias entre los grupos de baja y alta condición socioeconómica. También se efectuó la validez de contenido, y la medición de fiabilidad por medio de la consistencia interna y las fiabilidades inter e intra-observador y estabilidad prueba-reprueba. Adicionalmente se midió el acuerdo entre las respuestas de padres e hijos. Resultados: La consistencia interna fue adecuada (alfa de Cronbach 0,76-0,83). Los padres de niños con mejor condición socioeconómica tuvieron mejores puntuaciones en todas las dimensiones $(\mathrm{p}<0,05)$. Las puntuaciones fueron mejores entre niños sanos que entre enfermos. Como en otros estudios las mujeres tuvieron puntuaciones más bajas que los hombres, y los niños más altas que los adolescentes. Los coeficientes de correlación intraclase para la evaluación de la fiabilidad estuvieron por encima de 0,7 en casi todas las dimensiones (CCI 0,60,92 ), pero para el acuerdo entre las respuestas de padres e hijos no alcanzaron niveles aceptables $(\mathrm{CCI}<0,69)$. Conclusiones: KIDSCREEN-27 versión para padres, es un instrumento confiable y valido para ser utilizado en la medición de la calidad de vida en niños y adolescentes en el contexto colombiano.

\section{Calidad del sueño en una Facultad de Medicina de Lambayeque.}

Bartra Aguinaga A, Bendezú-Barnuevo D, Granados-Carrasco Z, HuamanchumoMerino J, Hurtado-Noblecilla E, Jiménez-Flores J, León-Jiménez F.

Escuela de Medicina de la Universidad Católica Santo Toribio de Mogrovejo, Chiclayo, Perú.

Antecedentes: La privación crónica de sueño genera alteraciones en el comportamiento, la atención, la memoria y el ánimo, equivalentes a permanecer tres días seguidos sin dormir. Los estudiantes universitarios y en especial los de ciencias de la salud tienen una elevada prevalencia de problemas del sueño. No se tienen datos locales al respecto. Objetivos: Determinar la frecuencia de mala calidad de sueño en una Facultad de Medicina de Lambayeque y comparar las frecuencias de mala calidad de sueño entre las escuelas de la Facultad durante Octubre del 2011. Material y métodos: Estudio transversal, analítico; muestreo probabilístico, aleatorio, estratificado, bietápico. Se empleó el Índice de calidad de sueño de Pittsburg, validado en español y en Perú. Se usó estadística descriptiva y para explorar una asociación entre mala calidad de sueño y otras variables, análisis bivariado mediante el $\mathrm{chi}^{2}$ y análisis multivariado mediante regresión logística. El proyecto fue aprobado por el comité de Bioética de la Universidad. Resultados: Se encuestaron 247 estudiantes: 194 mujeres (78,5\%) y 53 varones $(21,5 \%)$. La edad media fue de $20,04 \pm 2,5$ años. Hubo 210 "malos dormidores" ( $85 \%)$; la frecuencia de mala calidad de sueño según escuelas fue: medicina $(89,5 \%)$, enfermería $(86,4 \%)$, odontología $(84,4 \%)$ y psicología $(78,2 \%)$. El mayor uso de hipnóticos se reportó en psicología: $21,8 \%$. En el análisis bivariado sólo el uso de hipnóticos se asoció a mala calidad de sueño: Razón de prevalencias: 6,4 (IC 1-270,6; p: 0,039). En el multivariado, ninguna variable se asoció a mala calidad de sueño. Conclusiones: En esta Facultad de Medicina, existe alta frecuencia de mala calidad de sueño, la escuela con mayor uso de hipnóticos es Psicología y aparentemente en un análisis exploratorio inicial, no existe asociación entre mala calidad de sueño, la escuela, el sexo y la mala calidad "subjetiva" de sueño.

\section{Factores de riesgo asociados a la parasitosis intestinal en niños de una comunidad rural, Chiclayo - Perú, 2009.}

Malca-Tello N, Alvites-Castillo V.

Universidad Católica Santo Toribio de Mogrovejo; Chiclayo, Perú.

Objetivo: Identificar los factores de riesgo asociados a la parasitosis intestinal en niños de la comunidad rural Centro Poblado Pacherrez, Lambayeque, 2009. Metodología: Estudio descriptivo transversal. La población de estudio fueron los niños de 1 a 9 años del CP Pacherrez, durante 2009; para el diagnóstico de parasitosis se realizaron 03 veces para cada análisis coprológico por niño. Para la prevalencia de parasitosis (tipo de parásito y frecuencia) en los niños, los datos se reunieron en 4 grupos etarios: 1 a 2 años, 3 a 5 años, 6 a 7 años y 8 a 9 años, aplicándose después la prueba de hipótesis estadísticas de $\chi 2$, para estimar asociación sexo - edad. Se aplicó la prueba de hipótesis estadísticas de $\chi 2$ de Independencia de Criterios para la asociación de los factores de riesgo de la parasitosis. Resultados: La prevalencia de parasitosis en niños de 1 a 9 años fue del 49\%. El protozoario Giardia lamblia y Entamoeba coli fueron los parásitos más frecuentes $(57,4 \%$ y $25,1 \%$, respectivamente); en menor proporción se presentaron Enterobius vermicularis, Iodamoeba bütschlii, Blastocystis hominis e Hymenolepis nana. Los factores de riesgo que con mayor frecuencia se asocian a los casos de parasitosis intestinal infantil son: Ingreso económico familiar, educación de los padres, abastecimiento de agua (pozo y/o acequia), tienen "sitio" de lavado de manos, el no lavado de manos antes de las comidas, y refrigeración de los alimentos. Conclusiones: Los factores de riesgo asociados encontrados con respecto a la prevalencia de parasitosis intestinal en niños son las bajas condiciones socioeconómicas de la población, deficiente saneamiento sanitario y pobres hábitos de higiene. 
33. Validación de un instrumento para medir el nivel de conocimientos sobre hígado graso no alcohólico en médicos generales de Chiclayo.

Correa-Carhuachín V, Manayalle-Torres C, León-Jiménez F, Cubas-Benavides F, Díaz Vélez C.

Uniaz Vélez C.

Antecedentes: El hígado Graso no Alcohólico (HGNA) es una enfermedad prevalente en atención primaria. Puede evolucionar a cirrosis si no es detectado. Gran parte de los médicos de atención primaria no conocen el diagnóstico, tratamiento y pronóstico de esta patología. No existe un instrumento que mida el nivel de conocimiento sobre HGNA en médicos de atención primaria. Objetivo: Diseñar y validar un instrumento para determinar el nivel de conocimiento sobre HGNA en médicos generales de los distritos de Chiclayo y La Victoria. Material y métodos: Diseño de estudio: Validación de un instrumento. En base a la literatura, se diseñó un primer constructo de 13 preguntas; se analizó la validez de contenido mediante la valoración por 11 expertos según metodología Delphi. Se realizó una prueba piloto, entrevistando a 15 médicos generales de estos distritos. Se evaluó la fiabilidad del cuestionario analizando la consistencia interna por medio del alfa de Crombach y la fiabilidad test-retest mediante el coeficiente de correlación intraclase. La validez de constructo se llevó a cabo mediante el análisis factorial. Resultados: El alfa de Crombach fue de 0,77. La correlación intraclase tuvo un valor de 0,77 . El valor de la prueba de Kaiser - Meyer-Olkin fue de 0,091 y del test de esfericidad de Bartlett fue de 0,003; por lo que no se consideró pertinente llevar a cabo análisis factorial. Conclusiones: Se obtuvo un instrumento válido, con confiabilidad adecuada y de una sola dimensión para medir el nivel de conocimiento sobre hígado graso no alcohólico en médicos generales.
34. Validación de un instrumento para medir el nivel de conocimientos en médicos que laboran en emergencias sobre el manejo del Síndrome Isquémico Coronario Agudo.

Castañeda-Díaz $M^{1}$, Requelme-Portocarrero $F^{1}$, León-Jiménez $F^{1}$, Málaga-Rodríguez $G^{2}$, Díaz-Vélez $C^{1}$

${ }^{1}$ Universidad Católica Santo Toribio de Mogrovejo; Chiclayo, Perú.

${ }^{2}$ Universidad Peruana Cayetano Heredia; Lima, Perú.

Antecedentes: Los síndromes coronarios agudos (SICA) tienen elevada prevalencia mortalidad. Cuánto conocen los médicos en Lambayeque acerca de ésta patología, es incierto. Objetivo: diseñar y validar un instrumento para medir el nivel de conocimientos en médicos que laboran en emergencias en el manejo de SICA. Material y métodos: Tipo de estudio: validación de un instrumento. Se elaboró un constructo en base a las guías clínicas del American Heart Association y del American College of Cardiology de 17 preguntas: 9 de diagnóstico, 3 de pronóstico y 5 de tratamiento. La validación de contenido se basó en 2 procesos de validación por expertos. Se aplicaron dos pruebas piloto a residentes de Medicina Interna y médicos generales. La confiabilidad se determinó mediante el $\alpha$ de Cronbach. Se intentó realizar un análisis factorial mediante el cálculo de los valores de Kaiser Meyer Olkin (KMO) y la prueba de Barlett para determinar el número de dimensiones del instrumento. Se utilizó el software estadístico SPSS versión 15. El proyecto fue aprobado por el Comité de Bioética de la Universidad Santo Toribio de Mogrovejo y Comité de ética del Hospital Nacional Almanzor Aguinaga Asenjo; se obtuvo consentimiento informado de cada participante. Resultados: El $\alpha$ de Cronbach fue de 0,882; los valores de KMO y de la prueba de Barlett fueron no significativos por lo que no se pudo llevar a cabo el análisis factorial. Conclusiones: se obtuvo un instrumento con validación por expertos, confiabilidad aceptable y de una sola dimensión para medir el nivel de conocimientos sobre SICA en médicos que laboran en emergencias.

\section{Improving Care for Non-Heterosexuals: Pilot Validation of HIV and Health Care Algorithms for Men Who have Sex with Men (MSM) Populations in Latin- America and the Caribbean.}

Ceccarelli MA ${ }^{1}$, León S, Pérez-Luna $\mathbf{G}^{\mathbf{1}}$, Salvatierra J, Mazín $\mathbf{R}^{2}$, Cáceres $_{\mathbf{C}} \mathbf{C F}^{\mathbf{1}}$.
1 Unidad de Salud, Sexualidad y Desarrollo Humano, Universidad Peruana Cayetano Heredia.
2 Organización Panamericana de la Salud, Washington DC.

Background: Health service capacity to meet HIV and other health needs of MSM is still limited in Latin America and the Caribbean. The Pan American Health Organization led an effort to develop six algorithms to improve HIV and other care for them. We conducted a pilot study in Guatemala, Mexico and Peru to identify barriers and provide recommendations for implementation. Methods: In 2010, in 4 Guatemalan, 2 Mexican and 3 Peruvian Health Centers which agreed to participate and/or were identified by Ministries of Health, we applied a baseline interview to service managers, providers and users. Then health providers received 2-day training in algorithm use. During 6-month roll-out, health providers were asked to apply algorithms according to need, and complete individual clinical records with details of algorithms used, references made and difficulties found. Finally, endline semistructured interviews were conducted among providers and users. Results: We interviewed 13 service managers, 32 healthcare providers and 158 MSM users overall. Service managers stated that feasibility was related to extension of service hours, improvements in mental health services, drug supply, laboratory support, and training. After training in algorithm use, health providers reported they found the algorithms helpful, but more in-depth training was still needed. Most used algorithms were: HIV risk and infection, anorectal care, and sexual concerns. The anorectal care algorithm was perceived as the most innovative. Users perceived an improved provider-user relationship, and improved focus on HIV education, but stated the need of extended service hours and better diagnostic support for other infectious diseases. Conclusions: Improved guidance on HIV and comprehensive care for MSM is clearly needed in Latin America. Lack of trained human resources and mental health support should be addressed. These algorithms should be broadly disseminated to promote commitment for their adoption in the region, taking into account recommendations of this study.

\section{Psychometric properties of the SF-36 questionnaire.}

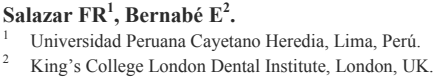

This study assessed the psychometric properties of the SF-36 questionnaire in a sample of 4344 Peruvian people aged 15 to 64 years. Internal consistency reliability was estimated using Cronbach's $\alpha$ coefficient, construct validity by known-groups comparison defined with respect to key sociodemographic characteristics, and factor structure by confirmatory factor analysis (CFA) at item level. Cronbach's $\alpha$ coefficient for the full questionnaire was 0.82 and ranged between 0.66 and 0.92 by scales. The 8 scales discriminated wel between sexes, age, and socioeconomic groups. CFA showed that a model with 8 first-order factors and 2 second-order factors (namely, physical and menta health) was a feasible representation of the SF-36 factor structure and had better fit to data than alternative factor structures. The SF-36 showed appropriate psychometric properties regarding internal consistency, construct validity, and factor structure when tested in Peru. Future studies should focus on testing other psychometric properties of the SF-36, such as convergent and discriminant validity, test-retest reliability, and sensitivity to change. 


\section{Factores asociados a la diabetes mellitus tipo 2 en la población de la provincia de Chiclayo. Junio a diciembre 2011.}

Leguía-Cerna JA, Morales-Cabrejos MA, Peña-Sánchez R, Díaz-Vélez C. Universidad Católica Santo Toribio de Mogrovejo; Chiclayo, Perú.

Antecedentes: Diabetes Mellitus es una enfermedad crónica del metabolismo de glucosa. Constituye una de las principales enfermedades crónicas que afecta nuestra población, siendo la diabetes Mellitus tipo 2 la de mayor prevalencia a nivel mundial y frecuente aparición en la etapa adulta, sobre todo en mayores de 40 años. Motivo por el que los investigadores nos planteamos determinar los factores asociados a la Diabetes Mellitus Tipo 2 en la población de Chiclayo durante junio - diciembre 2011. Material y métodos: Estudio Epidemiológico, Transversal, Analítico. La muestra estuvo constituida por 455 personas, con muestreo aleatorio estratificado, cuyos criterios de inclusión fueron: pobladores de Chiclayo de ambos sexos, mayores de 45 años, y de exclusión: neoplasias, enfermedades crónicas terminales o tratamiento cuyos efectos colaterales sean la alteración del metabolismo de la glucosa. Quienes aceptaron, llenaron una ficha con información general, factores de riesgo y hereditarios, así como la realización de una prueba rápida de glicemia capilar en ayunas. Las fichas se ingresaron a una base de datos utilizando el software Microsoft Excel XP. El procesamiento y análisis de datos se realizó mediante el programa estadístico SPSS v. 17, utilizándose ajustes por ponderación en cada estrato para las estimaciones puntuales. Resultados: La prevalencia de la diabetes mellitus 2 en Chiclayo es $15,6 \%$. El perfil epidemiológico predominante es el grupo etario entre 40-64 años, así mismo el sexo masculino, de procedencia urbana, grado de instrucción superior, y cuentan con seguro del estado. En características clínicas tienen los factores de herencia de Hipertensión Arterial, Diabetes Mellitus 2 y Dislipidemias; cuyo recurso terapéutico más empleado fueron secretagogos de insulina más sensibilizadores. Conclusiones: Las características clínicas asociadas fueron la herencia de Hipertensión Arterial, Diabetes Mellitus 2, dislipidemias asociados, sendetarismo y obesidad.

\section{Factores asociados al uso de herramientas en investigación en docentes universitarios.}

Díaz-Vélez C, León-Jiménez F, Peña-Sánchez R.

Universidad Católica Santo Toribio de Mogrovejo; Chiclayo, Perú.

Objetivo: Identificar los factores asociados al uso de herramientas de investigación en docentes de una universidad de Lambayeque. Material y Métodos: La base de datos del Censo Nacional Universitario 2010 se encuentra disponible en la página web de la Universidad Nacional Pedro Ruíz Gallo. El análisis de datos de fuentes secundarias y de conocimiento público (acceso en la página web: http://www.unprg.edu.pe/univ/modulos/informacion/index.php?sw=1\&tipo=no t\&id=282), no requirió la revisión y aprobación por comité de ética. Sin embargo, se enfatizó en confidencialidad, ningún nombre individual fuera utilizado, toda la información sería usada en estadísticas. La información está disponible en bases de datos del paquete estadístico SPSS. Resultados: Se usó cuestionario auto-aplicado en 760 docentes, el uso de biblioteca virtual fue Letras $(68,1 \%)$; uso de base de datos y software estadísticos en Ingeniería con $37,1 \%$ y $29,5 \%$, respectivamente. Las bases de datos más referidas fueron: en Ingeniería EBSCO: 30,3\%; Letras: EBSCO: 22,1\%; en Biológicas: SciELO $29,8 \% ; 73,42 \%$ habían asesorado al menos una tesis. El haber publicado en revista nacional y extranjera fue más frecuente en Biológicas $9,3 \%$ y $14,9 \%$, respectivamente. En el análisis multivariado se encontró como factores asociadas el uso de biblioteca virtual, capacitación docente (RP: 0,76; IC95\% 0,61-0,96) y realizar investigación (RP: 0,74, IC95\% 0,54-0,99). Los factores asociadas a uso de bases de datos bibliográficas fue pertenecer a Facultad de medicina (RP: 2,3 ; IC95\% 1,2-4,3), capacitación docente (RP: 0,76; IC95\% $0,6-0,96)$. Los factores asociados al uso de software estadísticos fue pertenecer a la facultad de Ciencias matemáticas y computación (RP: 2,1; IC95\% 1,133,92 ), facultad de ingeniería agrícola (RP: 2,36 ; IC95\% $1,14-4,88$ ) y facultad de Ingeniería civil sistemas y arquitectura (RP: 2,37; IC95\%1,25-4,49). Conclusiones: Los factores asociados al uso de biblioteca virtual (capacitación docente, realizar investigación), base de datos bibliográficas (pertenecer a facultad de medicina y capacitación docente) y software estadísticos (facultad ciencias matemáticas y computación, ingeniería agrícola e Ingeniería civil sistemas y arquitectura)

40. Necrosis papilar renal: Utilidad en el diagnóstico precoz de la urografía por tomografía computada vs la urografía endovenosa.

39. Análisis del Test de Morisky-Green-Levine como instrumento de medición en salud para estimar adherencia a tratamiento farmacológico en pacientes con enfermedades crónicas.

Monsalves MJ, Becerra A, Alvarado C.

Universidad de La Frontera; Temuco, Chile.

Antecedentes: Chile ha sufrido cambios desde el punto de vista de su estructura poblacional, causas de enfermedad y muerte. Esto ha producido un nuevo escenario que se ha traducido en cambios epidemiológicos significativos para el país. Actualmente, el escenario de salud nos centra en una población adulta que presenta altos niveles de morbilidad de enfermedades crónicas que requieren de intervenciones constantes de los equipos médicos. Contar con instrumentos que permitan medir la adherencia a estos tratamientos es de gran ayuda para el equipo medico y la constante vigilancia que demandan los pacientes polimedicados. Un ejemplo es el Test de Morisky-Green-Levine, recomendado por las guías clínicas del país como instrumento de medición de adherencia a tratamiento farmacológico. Objetivo: Realizar análisis del estado del arte en relación al Test de Morisky-Green-Levine (Test MGL) como instrumento de medición en salud para estimar adherencia a tratamiento farmacológico en pacientes con enfermedades crónicas. Material y métodos: Se realizó una revisión bibliográfica de los estudios que analizan el Test MGL. Se incluyeron estudios de validación y ensayos clínicos, en los últimos 20 años. Para la búsqueda se combinaron diferentes estrategias en las bases de datos de PubMed, SciELO, Cochrane y otros. Resultados: Se encontraron 8 estudios que analizan este Test, sin embargo ninguno de éstos describe el proceso de validación, ni profundizan en todas sus características psicométricas. La mayor parte de los estudios se presentan bajo un diseño de pruebas diagnósticas $(n=6 / 8)$. No existe concordancia entre estos estudios en cuanto a la especificidad y sensibilidad del test. Conclusiones: Es necesario realizar en un principio la revisión sistemática del tema y su posterior validación en Chile. Con el fin de certificar su aplicación en nuestra población como instrumento de medición, a través de un proceso que nos asegure confiabilidad y validez.
Quiñones F, Monsalves MJ.

Universidad de la Frontera; Temuco, Chile.

Antecedentes: La necrosis papilar renal representa un término descriptivo de condición multifactorial, en la que pueden coexistir múltiples etiologías. Dentro de las más conocidas encontramos las enfermedades crónicas con repercusión renal como la diabetes y el abuso de AINE. Entre las consecuencias inmediatas de esta patología, se describen el deterioro de la función renal y el síndrome nefrótico. Objetivo: Comparar la urografía por tomografía computada (UroTC) y la urografía endovenosa como método diagnóstico para la necrosis papilar renal. Material y métodos: Se realizó una revisión bibliográfica de los estudios que analizan el valor diagnóstico de la UroTC y la urografía endovenosa como método diagnóstico para la necrosis papilar renal. Se incluyeron estudios de los últimos 10 años en relación al tema. Se combinaron estrategias de búsquedas en Pubmed, SciELO y revistas especializadas. Resultados: La mayoría de los artículos revisados señalan que la necrosis papilar es una entidad clásica de la radiológica con una gran correlación imagenológica de sus diferentes patrones de evolución patológica, siendo similares éstos, tanto en la paleografía de eliminación como en la UroTC. Pero, esta última permite una detección precoz del cuadro renal. Conclusiones: Los antiguos métodos de imágenes se enfocaban en la detección de la causa desencadenante para evitar la progresión patológica. Sin embargo, actualmente, por medio de la UroTC se puede detectar en forma precoz lo que permite detener y eventualmente revertir la lesión, antes de que esta se establezca. Es recomendable que el clínico solicite por tanto, una UroTC ante la sospecha de esta patología con el fin de realizar una pesquisa temprana. 


\section{Rendimiento en la prueba saber 11 en personas con $y$ sin discapacidad.}

Padilla-Muñoz $\mathbf{A}^{1}$, Rodríguez $\mathbf{V}^{2}$, Castro $\mathrm{S}^{2}$, Reveiz $\mathbf{Y}^{2}$, Gómez-Restrepo $\mathbf{C}^{2}$.
Universidad del Rosario, Colombia.

Universidad del Rosario, Colombia.

Pontificia Universidad Javeriana, Colombia.

Introducción: La Convención de los Derechos de las Personas con Discapacidad (PCD) dispone que los Estados deben garantizar el acceso a la educación, sin ningún tipo de discriminación y manteniendo la igualdad de oportunidades. El estado colombiano ha estado interesado en la realización de pruebas estandarizadas para estudiantes PCD a través adaptaciones en el examen nacional. Objetivo: Comparar el rendimiento de la Prueba Saber 11 de PCD respecto a la cohorte de estudiantes sin discapacidad que asisten a la misma institución. Métodos: A través de un análisis secundario de tipo casocohorte se compararon los resultados en la prueba Saber 11 entre personas con y sin discapacidad dentro de cada institución educativa, para ello se estandarizaron los puntajes de los casos (PCD) con la cohorte de su misma institución (personas sin discapacidad), estableciendo las distancias en términos de desviaciones estándar del promedio obtenido por la persona con discapacidad contra el promedio de su cohorte en general. Se categorizó el puntaje en alto, medio y bajo y se calcularon los RR de PCD en cada categoría utilizando una regresión multinomial. Resultados: 529.651 estudiantes presentaron la prueba durante el 2009, $842(0,16 \%)$ reportaron tener algún tipo de discapacidad. El grupo de estudiantes sin discapacidad presenta un promedio mayor que el promedio de los estudiantes con discapacidad, siendo menores las diferencias en los invidentes. Adicionalmente se observa que los riesgos relativos (RR) de obtener un puntaje bajo dado que se tiene discapacidad, siempre es mayor que uno. Conclusión: Los estudiantes con discapacidad obtienen menores puntajes en las pruebas, equivalentes entre uno a tres años de educación, estas diferencias pueden ser porque los estudiantes son menos competentes o por que las pruebas aún no están lo suficientemente adaptadas, o una mezcla de ambos.

\section{3. ¿Dónde empieza el plagio de un artículo científico? Protocolo o informe final.}

Díaz-Vélez $\mathrm{C}^{1,2}$, Custodio-López $\mathbf{J}^{1,2}$, Díaz-Manchay $\mathbf{R}^{1,2}$, Poma-Ortiz $\mathbf{J}^{1,2}$, Taypicachuana-Juárez $\mathbf{J}^{1,2}$, Salazar-Ramírez $\mathrm{N}^{1,3}$, Colchado-Aguilar $\mathbf{J}^{1}$. ${ }^{1}$ Comité de Investigación del Hospital Nacional Almanzor Aguinaga Asenjo; Chiclayo, Perú. ${ }^{2}$ Docente de la Universidad Católica Santo Toribio de Mogrovejo; Chiclayo, Perú. ${ }^{3}$ Docente de la Universidad Señor de Sipán; Chiclayo, Perú.

Antecedentes: En el ámbito académico, el plagio puede ser deliberado (intencional) o inconsciente (por falta de conocimiento de lo que constituye plagio). "Aproximadamente el $80 \%$ de los estudiantes universitarios admiten que han plagiado". Objetivo: Estimar la frecuencia de plagio según característica de filiación y las páginas más usadas para plagio en protocolos de investigación presentados al comité de investigación de la Red Asistencial Essalud de Lambayeque 2011. Material y Métodos: La población fue 121 proyectos presentados, siendo excluidos los proyectos sin versión electrónica (06) y los ensayos clínicos presentados por sponsor externo (09), quedando 106. Se utilizó el software Viper antiplagiarism versión 1.5, analizando la versión virtual en formato doc y pdf contra el internet, para obtener la frecuencia de plagio. Luego que el software coloreara el párrafo se verificaba si presentaba su correspondiente llamado bibliográfico con el cambio de letra o encerrado entre comillas para delimitar que era una cita directa. Resultados: E1 $84,9 \%$ (90) fue de pregrado, 61,3\% (65) investigaciones de tipo cuantitativa, procedentes de USAT 42,5\% (45), USMP 34,9\% (37), UNPRG 17,9\% (19), otras 4,7\% (5), pertenecientes a facultad Medicina 61,3\% (65), enfermería $34,9 \%$ (37), diseño descriptivo $82,7 \%$ (87) y analítico $17,9 \% \quad(19)$. Obteniéndose $14,28 \% \pm 10,72$ en promedio de plagio fluctuando de $2 \%$ a $53 \%$. No más del $10 \%$ del escrito debe ser citas directas, es decir que el mejor de los casos el plagio sería de hasta 58,5\%. Páginas más usadas para plagio: http://www.monografias.com (28\%), http://apuntes.rincondelvago.com (23\%), http://es.wikipedia.org (20\%), http://www.alipso.com/ (12\%). Conclusiones: La frecuencia de plagio llega hasta por lo menos $58,5 \%$, siendo mayor en postgrado, investigación de tipo cualitativa, analítico y facultad de enfermería.

\section{Comparaciones indirectas versus comparaciones directas: evaluación de un caso en artritis reumatoide.}

González-Vacarezza N, Deminco A, Alemán A, Croci A, Pérez A.

División Evaluación Sanitaria, Ministerio de Salud Pública, Uruguay.

Antecedentes: La comparación indirecta es una metodología que permite comparar dos tratamientos que no han sido comparados cabeza a cabeza. Por ejemplo, si un tratamiento A fue comparado respecto a un tratamiento B (A vs $\mathrm{B})$, y otro tratamiento $\mathrm{C}$ fue comparado versus el $\mathrm{B}$, es posible comparar en forma indirecta A vs C. Objetivos: Determinar las diferencias de resultados entre una comparación directa (ensayo cabeza a cabeza) y una comparación indirecta. Métodos: Se identificaron estudios en pacientes con artritis reumatoide que comparen [abatacept + metotrexato vs infliximab + metotrexato] (Schiff 2008), y estudios que comparen [abatacept + metotrexato vs placebo + metotrexato] (Kremer 2006) e [infliximab + metotrexato vs placebo + metotrexato] (Westhovens 2006). Se determinaron los riesgos relativos (RR) y los intervalos de confianza de $95 \%$ (IC95\%) para las variables de eficacia ACR20/50/70. Resultados: Las comparación directa entre abatacept vs infliximab para ACR20/50/70 a 6 meses resultaron en (RR IC95\%): 1,12 $[0,95-1,33], \quad 1,09 \quad[0,83-1,44]$ y $0,81 \quad[0,48-1,37]$, respectivamente. La comparación indirecta resultó en $0,74[0,57-0,97], 0,70[0,43-1,14]$ y 1,09 [0,47-2,50]. Discusión: Los resultados presentan una discordancia en todas las variables evaluadas, encontrándose una diferencia significativa en el ACR20. Este resultado posiblemente se deba a que el estudio (Westhovens 2006) incluyó pacientes con una menor severidad de la enfermedad respecto a los restantes estudios. Conclusiones: Los resultados de esta metodología dependen en forma importante de la comparabilidad de las poblaciones de los estudios incluidos, además de otras variables. Si bien en algunas situaciones este tipo de comparaciones constituyen la mejor herramienta disponible, los resultados deben interpretarse con cautela para la toma de decisiones en salud.

\section{Eficacia y seguridad de la vitamina $E$ en el tratamiento de las enfermedades benignas de la mama.}

Inga-Lozada M, Leiva-Páucar LV, León-Jiménez FE, Marcelo-Fuster L. Egresados de la Maestría de Epidemiología Clínica 2010 - 2011, Universidad Peruana Cayetano Heredia.

Antecedentes: Las enfermedades benignas de la mama (BBD) constituyen un grupo heterogéneo de patologías relacionadas a cambios fibroquisticos y a una diversidad de síntomas y signos como mastalgia, nodularidad, edema y eritema. La mastalgia es el síntoma más común; puede ser severa e interferir con las actividades de la vida diaria; el tratamiento óptimo no está aún definido. Diversos tratamientos han sido probados en pacientes con mastalgia, siendo la vitamina E uno de los más usados. Objetivo: Evaluar la evidencia clínica existente de eficacia y seguridad de la vitamina E comparado con placebo en el tratamiento de las enfermedades benignas de la mama. Material y métodos: Se realizó búsquedas en el registro de Cochrane Central de Ensayos controlados, MEDLINE (1980-Octubre 2011), LILACS (1980-Octubre 2011) y TRIPDATABASE; además en las bases de datos: REPEBIS, BIREME y REDALYC. Dos revisores seleccionaron y evaluaron de manera independiente los títulos, resúmenes, así como la calidad metodológica de cada ensayo. Se incluyeron ensayos clínicos doble ciego, aleatorizados, placebo controlado; se excluyó a los pacientes con Cáncer de mama y Síndrome premenstrual. El outcome de eficacia fue: a) Disminución de la intensidad del dolor según escala análoga visual. Para seguridad: a) Eventos gastrointestinales: náuseas, vómitos y diarrea. b) Aumento de transaminasas. Resultados: Sólo cumplieron los criterios de inclusión, 3 ensayos; si se consideró la variable disminución del dolor como numérica la heterogeneidad fue de $0 \%$, pero no hubo diferencia significativa con el grupo placebo. (DEM: 0,04 IC95\%: 0,15-0,23 p=0,68). La variable disminución del dolor como categórica, presentó una alta heterogeneidad $\left(\mathrm{I}^{2}: 57 \%\right)$ entre los estudios; no hubo diferencia significativa con el grupo placebo (RR: 1,27 IC95\%:0,81-1,98, p=0,3) a dosis de $150 \mathrm{y} / \mathrm{o}$ 300UI/día de Vitamina E. En ningún estudio se evaluó seguridad de la Vitamina E en las BBD. Conclusiones: No existe evidencia para afirmar que la Vitamina E sea útil en disminuir la intensidad del dolor en las BBD; en ninguno de los estudios se evaluó la seguridad de la vitamina E. 